\title{
Detecting Impacts of Proton Beams on the LHC Collimators With Vibration and Sound Measurements
}

\author{
O. Aberle, R. Assmann, A. Masi, S. Redaelli, G. Spiezia \\ CERN, Geneva, Switzerland
}

\begin{abstract}
The 360 MJ stored energy of the $7 \mathrm{TeV}$ LHC beams can seriously damage the beam line elements in case of accidental beam losses. Notably, the collimators will sit at 6 to 7 sigmas from the beam centre (1.2 to 1.4 $\mathrm{mm}$ ) and will be hit and possibly damaged in case of failures, with a consequent degradation of the cleaning performance of the overall collimation system. The experience from operating machines shows that an a-posteriori identification of the damaged collimators from the observed performance degradation is extremely challenging. Collimator tests with beam at the SPS have shown that the impact of $450 \mathrm{GeV}$ proton beams at intensities from $10^{10}$ to $3 \times 10^{13}$ could be detected by measuring sound and vibrations induced by the impacting beams.
\end{abstract}

CERN,

CH-1211 Geneva 23,

Switzerland

Geneva, June 2005 


\title{
DETECTING IMPACTS OF PROTON BEAMS ON THE LHC COLLIMATORS WITH VIBRATION AND SOUND MEASUREMENTS
}

\author{
S. Redaelli, O. Aberle, R. Aßmann, A. Masi, G. Spiezia, CERN, Geneva, Switzerland
}

\begin{abstract}
The $360 \mathrm{MJ}$ stored energy of the $7 \mathrm{TeV}$ LHC beams can seriously damage the beam line elements in case of accidental beam losses. Notably, the collimators will sit at 6 to 7 sigmas from the beam centre (1.2 to $1.4 \mathrm{~mm}$ ) and will be hit and possibly damaged in case of failures, with a consequent degradation of the cleaning performance of the overall collimation system. The experience from operating machines shows that an a-posteriori identification of the damaged collimators from the observed performance degradation is extremely challenging. Collimator tests with beam at the SPS have shown that the impact of $450 \mathrm{GeV}$ proton beams at intensities from $10^{10}$ to $3 \times 10^{13}$ could be detected by measuring sound and vibrations induced by the impacting beams.
\end{abstract}

\section{INTRODUCTION}

The LHC operation will require a powerful collimation system to clean beam halos and prevent quenches of the superconducting magnets. The system will consist of approximately 65 collimators per beam dedicated to the beam cleaning $[1,2]$. The collimator jaws will be centered around the circulating beam with gaps at $7 \mathrm{TeV}$ as small as $1 \mathrm{~mm}$ ( 6 beam sigmas). These are the closest elements to the beam and in case of accident failures will be hit and possibly damaged. The experience with presently operating hadron colliders shows that the diagnostics of damaged collimators after accidents is not easily possible. The online detection of beam impacts will certainly be helpful for the complex LHC collimation system.

Sound and vibration measurements have been proposed as a possible tool to detect impacts of high intensity proton beams on the LHC collimators. An experiment has been carried out during the collimator robustness test of November 2004 with $450 \mathrm{GeV}$ proton beams from the CERN SPS. An LHC collimator prototype has been equipped with high resolution accelerometers and a microphone to measure sound and vibrations induced by the impacting beams with different intensities and depths inside the collimator jaws. In this paper, the development of the data acquisition system and the measurement results are discussed.

\section{EXPERIMENTAL SETUP}

\section{Installation layout and measurement sensors}

The collimator robustness test was carried out at the TT40 tunnel, in the TI2 transfer line from the SPS to

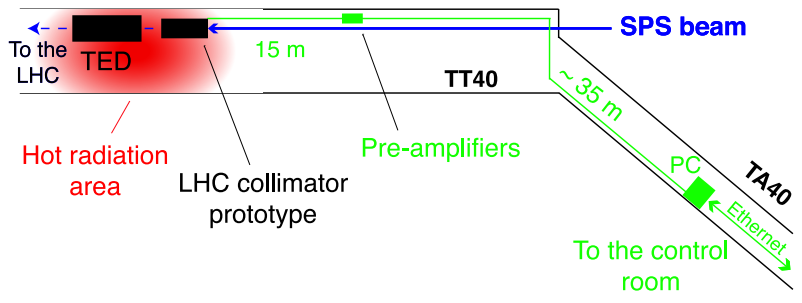

Figure 1: Installation layout for the collimator at TT40.

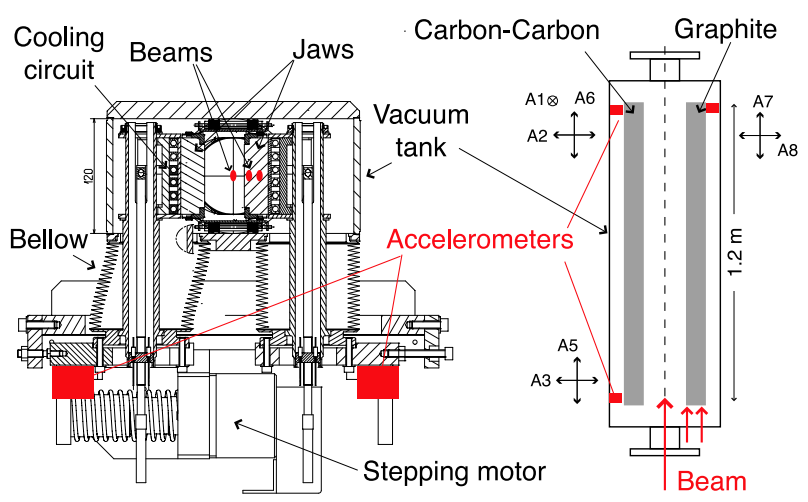

Figure 2: Front and top view of the collimator prototype.

the LHC. The installation layout is shown in Fig. 1. A fully operational prototype of the LHC secondary collimator [3] was installed a few metres upstream of a beam dump (TED). The collimator front and top view, with the locations of the vibration sensors, are given in Fig. 2. The collimator prototype was equipped with 7 piezoelectric accelerometers. They were installed such as to measure vibrations at different locations and along different orientations. These sensors were installed on the collimator support plate which transmits the motion from the stepping motors to the jaw through the jaw support bars. This is the closest location outside the vacuum that ensures a direct mechanical contact with the collimator jaws. In addition, a microphone was installed at approximately $1 \mathrm{~m}$ distance upstream of the collimator with respect to the in-

Table 1: Technical specifications of the used sensors.

\begin{tabular}{|l|c|c|c|}
\hline Sensor type & Num. & $\begin{array}{c}\text { Sensitivity } \\
{\left[\mathrm{pC} / \mathrm{m} / \mathrm{s}^{2}\right]}\end{array}$ & $\begin{array}{c}\text { Bandwidth } \\
{[\mathrm{Hz}]}\end{array}$ \\
\hline Acc. B\&K 2273AM1 & 1 & 1.1 & $0.1-6000$ \\
Acc. B\&K 2273A & 2 & 0.4 & $0.1-10000$ \\
Acc. AP Tech 40 & 2 & 2.1 & $0.5-10000$ \\
Acc. AP Tech 37 & 2 & 1.0 & $0.5-20000$ \\
\hline Micr. B\&K 4189 & 1 & $41.6[\mathrm{mV/Pa}]$ & $6.3-20000$ \\
\hline
\end{tabular}


Table 2: Parameters of the SPS beam

\begin{tabular}{|l|c|}
\hline Beam energy $[\mathrm{GeV} / c]$ & 450 \\
Bunch spacing $[\mathrm{ns}]$ & 25 \\
Beam intensity $\left[10^{12} \mathrm{p}\right]$ & $7.9-32$ \\
Beam power $[\mathrm{kW}]$ & $0.6-2.4$ \\
Beam size, $\mathrm{H} \times \mathrm{V}[\mathrm{mm} \times \mathrm{mm}]$ & $\approx 0.7 \times 1.2$ \\
Beam depths in the jaw $[\mathrm{mm}]$ & $1-6$ \\
\hline
\end{tabular}

coming beam direction. The main technical specifications of the various sensors are listed in Table 1. Radiation hard piezoelectric accelerometers by two manufacturers $B \& K$ and AP Tech have been used. The measurable frequencies are between $0.5 \mathrm{~Hz}$ and $10 \mathrm{kHz}$ and the sensitivities range from $0.4 \mathrm{pC} / \mathrm{m} / \mathrm{s}^{2}$ to $2.2 \mathrm{pC} / \mathrm{m} / \mathrm{s}^{2}$. For sound measurements a pre-polarized free-field microphone by $\mathrm{B} \& \mathrm{~K}$ was used. This is not a radiation hard device because it contains a preamplifier. Nevertheless, it was estimated that it should withstand the expected radiation doses at TT40. In order to protect sensitive electronics from radiation, the sensor pre-amplifiers were installed $15 \mathrm{~m}$ upstream of the collimator. A remotely controlled PC for the data acquisition was placed in the TA40 access tunnel to TT40, outside the high radiation area.

\section{The data acquisition system}

The signals from accelerometers and microphone are converted by conditioning pre-amplifiers into low impedance signals and transported to a PC with shielded coaxial cables. The sensors by B\&K are connected to a 4channel NEXUS pre-amplifier, which allows adjusting the output gains over 6 orders of magnitude, setting the output sensitivity of the transducers and changing low/high pass filters for each channel independently. These functionalities are remotely controlled via RS232 connection from the PC. The AP Tech accelerometers used independent fixedgain amplifiers. For both systems, $35 \mathrm{~m}$ long coaxial cables were used to transmit the signal to a PC, located in a low radiation area (see Fig. 1). The measured signals were acquired with an 8-channel, Sigma-Delta ADC card by National Instruments, model PCI-4472, integrated on the PC. All channels are simultaneously sampled at acquisition frequencies up to a $102.4 \mathrm{kHz}$ and have a nominal resolution of 24 bit over an input range of \pm 10 Volt. Typically, data were acquired at a sampling rate of $51.2 \mathrm{kHz}$ to satisfy the Nyquist criterium for all devices (see Table 1).

The control of the data acquisition board and of the NEXUS amplifier (via RS232) were performed with dedicated application software developed in LabWindow 7.0®. The data acquisition at high sampling rate is performed with a dynamic circular buffer. The software also allows an on-line display and a post-processing Fourier analysis of the measured data. The PC, unaccessible throughout the tests with beam, was controlled from the control room via a Remote Desktop ${ }^{\circledR}$ application under the Windows XP® operating system.

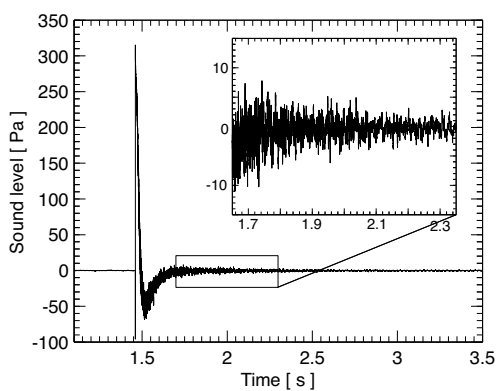

Figure 3: Microphone signal versus time as measured during a $3 \times 10^{13}$ proton beam impact on the collimator jaw.

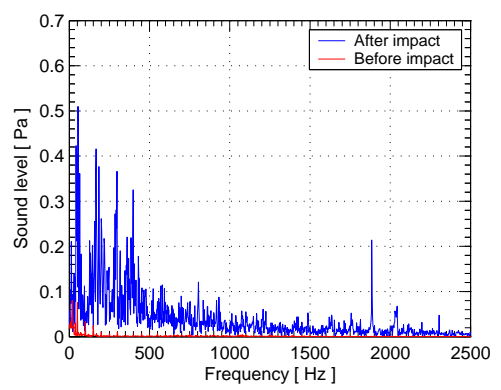

Figure 4: Fourier amplitude of the microphone signal before (red) and after (blue) a $3 \times 10^{13}$ proton beam impact.

This solution with DAQ system inside the tunnel was adopted to avoid transmitting the measured signals over distances of $\approx 150 \mathrm{~m}$ from the collimator location to the closest accessible area. In order to increase the reliability of the system, a remote switch based on an Internet web server was installed and used to remotely force the reboot of the PC in case of unrecoverable system failures. These were indeed encountered during the test with beams.

\section{Experimental conditions}

The beam parameters at the collimator are summarized in Table 2. Measurements have been performed for both jaws in two conditions: (1) with increasing beam intensities at fixed beam impact depth of $5 \mathrm{~mm}$ from the jaw surface (see Fig. 2) and (2) with beam impact depths from $1 \mathrm{~mm}$ to $6 \mathrm{~mm}$ at a beam intensity of $3.2 \times 10^{13}$ protons.

\section{MEASUREMENT RESULTS}

\section{Sound measurements}

Fig. 3 shows an example of a microphone signal acquired when a full LHC injection batch of $3 \times 10^{13}$ protons (stored energy of $2.4 \mathrm{~kW}$ ) impacts on the carbon-carbon collimator jaw. The signal is characterized by a sudden spike synchronized to the beam passage, followed by an exponential decay with typical time constants of $\approx 300 \mathrm{~ms}$. There are indications that this spike could be induced by radiation effects. Nevertheless, the sensor recovers after $\approx 350 \mathrm{~ms}$. The spike does not significantly contribute to the sound but just results in a sound distortion. The sound from the colli- 


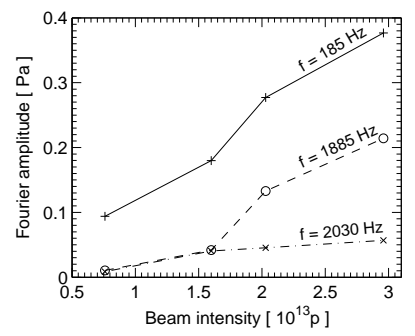

Figure 5: Fourier amplitude of three different frequencies versus beam intensity as measured with the microphone.

mator is different than what is recorded when beams impact on the TED and this proves the potential of using sound measurements to detect beam impacts.

The Fourier analysis of the sound signal is shown in Fig. 4. The frequency content of the noise before the beam impact (red line) is compared with the one calculated $\approx 400 \mathrm{~ms}$ after the spike (blue line). A fast Fourier transform is calculated over a sample of $400 \mathrm{~ms}$ (frequency resolution of $2.5 \mathrm{~Hz}$ ). The beam impact excites a number of frequencies in a broad frequency range that extends up to approximately $4 \mathrm{kHz}$. For example, large amplitude peaks are found at $168 \mathrm{~Hz}, 185 \mathrm{~Hz}, 430 \mathrm{~Hz}, 1185 \mathrm{~Hz}$ and $2030 \mathrm{~Hz}$. The amplitudes of these peaks increase with the intensity of the impacting beam, see Fig. 5. It is interesting to note that higher intensity beams do not induce different vibration frequencies, as could be expected in case of damage. This is consistent with the inspections after the beam tests that showed no major damage to the collimator jaw.

In Fig. 6, the RMS amplitudes of the microphone signals are given as a function of the beam intensity (left) and of the beam impact depth (right), which is defined as the distance from the beam centre to the jaw surface (see Fig. 2). The RMS values are calculated on a $2.5 \mathrm{~s}$ time window, starting $400 \mathrm{~ms}$ later than the beam passage. A clear dependence on beam energy and impact depth is noticed. The RMS amplitude of the microphone signal increases linearly with the beam intensity over the full range from $0.7 \times 10^{13}$ to $3.0 \times 10^{13}$ protons. A linear increase is also measured for impact depths up to $3 \mathrm{~mm}$, i.e. 5 horizontal beam sigmas, and a saturation is found for larger depths.

\section{Accelerometric measurements}

A typical accelerometer signal as measured with an impacting beam of $3 \times 10^{13}$ protons is shown in Fig. 7 (left). The signal shows a sudden rise and an exponential decay. Unlike for the microphone, the vibration signal after decay lasts for a shorter time, which makes it more difficult to perform a Fourier analysis. An example is shown in Fig. 7 right part. Accelerometers were sensitive to a $50 \mathrm{~Hz}$ electromagnetic noise. The spectrum shows a number of excited frequencies in the same frequency range as it was observed in the sound data. In some cases, the frequency peaks are slightly shifted with respect to the microphone data. For instance, peaks at $417 \mathrm{~Hz}, 1965 \mathrm{~Hz}$ and $2045 \mathrm{~Hz}$ are found. The measured frequencies are being cross-checked with the expected of mechanical vibrations
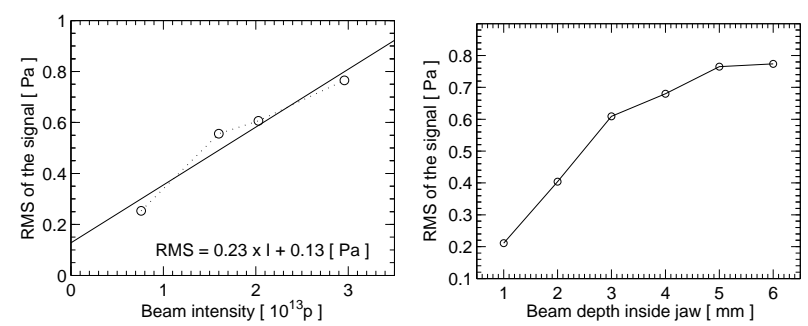

Figure 6: RMS amplitude of sound signal versus beam intensity (left) and beam impact depths inside the jaw (right).
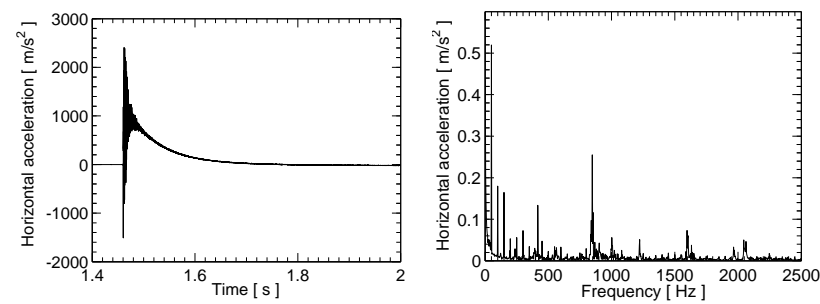

Figure 7: Time (left) and frequency (right) accelerometer signal for impacting beam intensity of $3 \times 10^{13}$ protons.

from a finite element model of the collimator. The radiation effects are under investigation with the manufacturer.

\section{CONCLUSIONS}

Sound and vibration measurements have successfully been used to detect impacts of high intensity, $450 \mathrm{GeV}$ proton beams on a LHC collimator prototype. Measurements at different beam intensities and beam impact depths show increasing sound levels and are in qualitative agreement with the expectations. Vibration measurements after beam tests did not indicate significant changes of the collimator jaw mechanical structure, which is in agreement with visual inspections of the prototype. The measurement system can in principle be used at the LHC to detect beam impacts and, hence, possibly damaged collimators. A better understanding of the sensor systematics in presence of instantaneous radiation bursts, in particular the effect on the transmission cable, and a optimization of the system architecture would be required for the usage in the LHC.

The authors would like to acknowledge all the colleagues who helped with stimulating discussions and in the experiment setup. In particular, G. Arduini, A. Bertarelli, L. Bottura, F. Cennamo, U. Cesaro, E. Chiaveri, A. Dallocchio, F. Decorvet, J.F. Deheurle, B. Dehning, M. Eller, A. Fabich, A. Ferrari, P. Françon, F. Loprete, M. Magistris, R. Perret, P. Sievers, R. Wilfinger, J. Wenninger.

\section{REFERENCES}

[1] O. Brüning (Ed.) et al, "LHC design report. Vol. I: The LHC main ring," CERN-2004-003 (2004).

[2] R. Aßmann et al., these proceedings.

[3] A. Bertarelli et al., "The mechanical design of the LHC collimators," EPAC04, Lucerne, Switzerland (2004). 